\title{
Development of c-MET-specific chimeric antigen receptor-engineered natural killer cells with cytotoxic effects on human liver cancer HepG2 cells
}

\author{
BING LIU ${ }^{1,2^{*}}$, ZHENG-ZHI LIU ${ }^{3 *}$, MEI-LING ZHOU ${ }^{1,2}$, JIAN-WEI LIN $^{1,2}$, XUE-MEI CHEN $^{1,2}$, \\ ZHU LI ${ }^{1,2}$, WEN-BIN GAO ${ }^{1}$, ZHEN-DONG YU ${ }^{4}$ and TAO LIU ${ }^{1,2}$ \\ ${ }^{1}$ Department of Biotherapy and Oncology; ${ }^{2}$ Public Service Platform for Cell Quality Testing and \\ Evaluation of Shenzhen, Shenzhen Luohu People's Hospital, Shenzhen, Guangdong 518001; ${ }^{3}$ Department of \\ Laboratory, Women and Children Health Institute of Futian, Shenzhen, Guangdong 518045; ${ }^{4}$ Central Laboratory, \\ Peking University Shenzhen Hospital, Shenzhen, Guangdong 518036, P.R. China
}

Received February 25, 2019; Accepted June 25, 2019

DOI: $10.3892 / \mathrm{mmr} .2019 .10529$

\begin{abstract}
In recent years, cellular immunotherapy has served an important role in the combined treatment of hepatocellular carcinoma. The possibility of specific cell therapies for the treatment of solid tumours has been further explored following the success of chimeric antigen receptor (CAR)-T cell therapy in the treatment of haematological tumours. The present study aimed to evaluate the specificity and efficiency of c-MET-targeted CAR-NK cell immunotherapy on human liver cancer in vitro. A CAR structure that targeted and recognised a c-MET antigen was constructed. c-MET-CAR was transferred into primary NK cells using lentiviral infection. c-MET-positive HepG2 cells were used as an in vitro study model. The cytotoxicity assay results revealed that c-MET-CAR-NK cells exhibited more specific cytotoxicity for HepG2 cells with high c-MET expression compared with the lung cancer cell line H1299, which has low levels of c-MET expression. The results of the present study demonstrated that
\end{abstract}

Correspondence to: Dr Tao Liu, Department of Biotherapy and Oncology, Shenzhen Luohu People's Hospital, 47 Youyi Road, Luohu, Shenzhen, Guangdong 518001, P.R. China

E-mail: tao2020@sohu.com

Mr. Zhen-Dong Yu, Central Laboratory, Peking University Shenzhen Hospital, 1120 Lianhua Road, Futian, Shenzhen, Guangdong 518036, P.R. China

E-mail: dongboyaa@163.com

${ }^{*}$ Contributed equally

Abbreviations: NK, natural killer; c-MET, c type proto-oncogene receptor tyrosine kinase; $\mathrm{CAR}$, chimeric antigen receptor; LDH, lactate dehydrogenase; HCC, hepatocellular carcinoma; PBMCs, peripheral blood mononuclear cells

Key words: NK cells, c-MET, chimeric antigen receptor, liver cancer, immunotherapy
c-MET may be a specific and effective target for human liver cancer cell CAR-NK immunotherapy. Based on these results, CAR-NK cell-based immunotherapy may provide a potential biotherapeutic approach for liver cancer treatment in the future.

\section{Introduction}

In China, the third most common cause of cancer-related mortality is hepatocellular carcinoma (HCC) (1). HCC accounts for $\sim 90 \%$ of the total liver cancer burden globally (2). In clinical practice, surgical resection is the most commonly used treatment procedure, followed by adjuvant and systemic chemotherapy. However, the complex aetiology and high metastatic potential of the disease renders surgical treatment futile in the majority of cases. In recent years, cellular immunotherapy has been increasingly applied in the treatment of HCC (3). Following the success of the new generation of targeted cell therapy technologies represented by chimeric antigen receptor (CAR)-T cytotherapy for the treatment of haematological tumours, researchers have begun to explore the possibility of specific cell therapies for the treatment of solid tumours (4). Currently, one of the major barriers to CAR-T cell therapy is cytokine release syndrome (CRS), which is mainly mediated by interleukin-6. CRS can lead to acute respiratory distress syndrome and multiple organ failure (5). Unlike $\mathrm{T}$ cells, natural killer (NK) cells mainly secrete interferon $\gamma($ IFN- $\gamma$ ) and granulocyte-macrophage colony-stimulating factor (GM-CSF), which are unlikely to cause CRS $(6,7)$. Therefore, CAR-expressing NK (CAR-NK) cells may be safer compared with CAR-T cells in clinical application.

NK cells, which are a crucial component of the innate immune system, are characterized as CD3- ${ }^{-}$D56 $6^{+}$and serve important roles in the immune surveillance and early control of tumorigenesis (8). NK cells can recognize and eradicate tumour cells without prior antigenic exposure $(9,10)$. Their recognition of tumour cells depends on the imbalance of activatory and inhibitory receptors on the cell surface (11). Following the identification of tumour cells, NK cells can destroy tumour 
cells rapidly via secretion of perforin and granzymes $(12,13)$, induction of apoptosis, expression of tumour necrosis factor family molecules (14) or expression of CD16, which leads to antibody-dependent cellular cytotoxicity $(15,16)$.

However, the anti-tumour activity of NK cells in patients with tumours is limited due to the decline in the quantity and quality of NK cells and tumour immune escape (9). Modifying NK cells through recombinant CAR engineering may enhance their tumour targeting and killing efficiency (17). CAR-NK cells have been tested in vitro and in animal models (6), but clinical studies of CAR-NK cells remain mostly in the preclinical stage. CAR-NK cells take advantage of the CAR gene-modification strategy and present several unique advantages over CAR-T cells (7). First, long-term persistence of CAR-expressing cells increases the risk of autoimmunity or malignant transformation; however, since NK cells have relatively limited lifespans in vivo, infused CAR-NK cells perish rapidly after mediating their antitumor effects. Second, allogeneic NK cells do not induce graft-versus-host disease (18). In addition, even when tumour cells escape immune surveillance by downregulating the expression of CAR-targeted antigens, CAR-NK cells are still be able to mediate cytotoxic effects through their natural receptor molecules (19). Notably, the cytokines secreted by NK cells are mainly the aforementioned IFN- $\gamma$ and GM-CSF, which are unlikely to cause CRS $(6,7)$. Considering the multiple advantages of CAR-NK cells, the potential applications of CAR-NK cells for the treatment of various cancer types are being studied widely (18).

A CAR construct consists of an extracellular antigen -recognition domain, a transmembrane domain and an intracellular signalling domain. The extracellular antigen-recognition domain is generally composed of a single-chain variable fragment ( $\mathrm{scFv}$ ) derived from the variable regions of the heavy and light chains of a monoclonal antibody, which are fused together via a flexible linker (19). The intracellular signalling region, which contains an immunoreceptor tyrosine-based activation motif, determines the intensity of CAR-NK activation (19).

Similar to T cells, NK cells can be gene-modified with CARs and specifically combined tumour antigens to enhance cytotoxicity (18). However, to the best of our knowledge, only a few previous studies have explored their therapeutic potential in HCC. Primary NK cells are usually derived from autogenous peripheral blood mononuclear cells (PBMCs) from patients (7). Primary NK cells were also examined for a comprehensive insight into NK cell-based modification (7). The c-MET-specific CAR in the present study was based on the humanized c-MET-specific antibody developed in our laboratory and the TYRO protein tyrosine kinase-binding protein (DAP12) signalling domains.

$\mathrm{c}-\mathrm{MET}$ is the product of the proto-oncogene $M E T$, which is expressed by epithelial and endothelial cells, neurons, hepatocytes and haematopoietic cells $(20,21)$. c-MET serves crucial roles in the development and progression of cancer (22). c-MET is associated with the proliferation, survival, invasion and metastasis of cancer cells (23). The overexpression of c-MET has been observed in various solid malignancies, such as liver (24-26), breast (27), lung (28) and colorectal (29) cancer. Notably, c-MET aberrations occur in $\sim 50 \%$ of patients with HCC (30). c-MET has been identified as a carcinogen in HCC, as increased c-MET activity can initiate, drive or contribute to the development and progression of HCC (25). Thus, c-MET has been increasingly used as an immunotherapeutic CAR-T cell target in progressive HCC. However, the lack of specific tumor antigens and the limited penetration of CAR-T cells into the tumour site reduce the effect of CAR-T cells on hepatocellular carcinoma (31).

In the present study, the function and effectiveness of c-MET-CAR-engineered NK cells against liver cancer cells was determined with the aim of developing an effective and specific NK cell-based therapeutic strategy for liver cancer treatment.

\section{Materials and methods}

Cell lines. The human liver cancer cell line HepG2 and lung cancer cell line H1299 (Shanghai Zhong Qiao Xin Zhou Biotechnology Co., Ltd.) were maintained in RPMI 1640 medium (Thermo Fisher Scientific, Inc.) supplemented with $10 \%$ fetal bovine serum (FBS; Thermo Fisher Scientific, Inc.). 293T cells (American Type Culture Collection) were cultured in high-glucose DMEM (Thermo Fisher Scientific, Inc.) containing $10 \%$ FBS. All cell lines were cultured in a humidified atmosphere containing $5 \% \mathrm{CO}_{2}$ at $37^{\circ} \mathrm{C}$.

RNA interference of Hep 62 cells. HepG2 cells were transfected with $100 \mathrm{nM}$ C-MET-homo-3918 and C-MET-homo-2659 short interfering (si) RNAs (Shanghai GenePharma Co., Ltd.) using Lipofectamine ${ }^{\circledR} 3000$ (Thermo Fisher Scientific, Inc.) according to the manufacturer's instructions. Scrambled siRNA was used as a negative control. Cells were cultured for $48 \mathrm{~h}$ prior to further experiments. To avoid off-target effects, two pairs of siRNAs were used for RNA interference: C-MET-homo-3918 forward, 5'-CCAGAGACAUGUAUGAUAATT-3' and reverse, 5'-UUAUCAUACAUGUCUCUGGTT-3'; C-MET-homo-2659 forward, 5'-GCAACAGCUGAAUCUGCAATT-3' and reverse, 5'-UUGCAGAUUCAGCUGUUGCTT-3'. The sequences of control siRNAs (scrambled negative control) were forward, 5'-UUCUCCGAACGUGUCACGUTT-3' and reverse, 5'-ACG UGACACGUUCGGAGAATT-3'.

Construction of the c-MET-CAR lentiviral plasmid. The scFv nucleotide sequence of an anti-c-MET antibody was fused with a sequence encoding truncated human epidermal growth factor receptor (huEGFRt) immediately following the V5 tag-encoding sequence. The fused DNA sequences were incorporated with 41BB-DAP12. The entire c-MET-scFv-CD8 $\alpha$-41BB-DAP12-EGFRt-V5 fragment was ligated into a green fluorescent protein (GFP)-expressing lentiviral vector Plv-Easy-GFP [previously constructed in our laboratory (32)] to construct the c-MET-CAR lentiviral plasmid. The plasmid was digested with the KpnI and NotI enzymes to confirm that the insert was the correct size. Sequencing analysis of the plasmid was completed by Sangon Biotech (Shanghai) Co., Ltd. An empty GFP lentiviral plasmid was used as a negative control.

Lentivirus production. To produce lentiviruses for the infection of NK cells, $293 \mathrm{~T}$ cells $\left(5 \times 10^{6}\right.$ cells/dish) were seeded in $10 \mathrm{~cm}$ dishes and cultured overnight. The c-MET-CAR 
lentiviral plasmid or GFP lentiviral plasmid was transfected with the psPAX2 and pMD2.G packaging plasmids (gifts from Professor Hu Ying) using a calcium chloride transfection reagent as previously described (33), the cells were incubated at $37^{\circ} \mathrm{C}$ with $5 \% \mathrm{CO}_{2}$ overnight and then the culture medium was replaced with fresh cell culture medium. The c-MET-CAR and GFP lentiviruses were harvested 48-72 h following transfection, filtered through Millex-GP $0.45 \mu \mathrm{m}$ filters (Merck KGaA), and concentrated by ultracentrifugation $\left(4^{\circ} \mathrm{C} ; 72,000 \mathrm{x} \mathrm{g} ; 2 \mathrm{~h}\right)$. The pellet was resuspended with $1 \mathrm{X}$ PBS. The c-MET-CAR lentivirus titer was determined by detecting GFP expression using a flow cytometry-based method. Briefly, 293T cells were plated at $1 \times 10^{5}$ cells/well in a 24-well plate and incubated overnight. The next day, the medium was removed and the cells were infected with $1 \mathrm{ml}$ of undiluted, 1:4-, 1:16-, 1:64-, 1:128- or 1:256-diluted c-MET-CAR lentivirus containing $8 \mu \mathrm{g} / \mathrm{ml}$ polybrene (Sigma-Aldrich; Merck KGaA). Following incubation overnight at $37^{\circ} \mathrm{C}$, the medium was replaced with fresh DMEM. At $48 \mathrm{~h}$, the cells were detached with trypsin in EDTA, resuspended in $1 \mathrm{X}$ PBS and analysed for GFP expression by flow cytometry. The titer of the c-MET-CAR lentivirus was calculated as follows: Titer $($ transducing units $/ \mathrm{ml})=($ percentage of $\mathrm{GFP}^{+}$cells $\left.\mathrm{x} 10,000\right) /$ dilution ratio.

Transduction of NK cells. Human PBMCs were isolated from fresh blood. Peripheral blood samples from donors were collected at Shenzhen Luohu People's Hospital (from June 2018 to January 2019). Written informed consent was collected and the study was approved by the Ethics Committee of Shenzhen Luohu People's Hospital. A Human NK Cell Culture kit (cat. no. MCF-004; Morecell Biomedical Co., Ltd.) and Serum-free Medium for NK Cells (cat. no. MCM-002; Morecell Biomedical Co., Ltd.) was used for the induction of NK cells, according to the manufacturer's instructions. The cell number and viability were determined under a microscope using trypan blue.The purity of CD $3^{-} \mathrm{CD}^{-} 6^{+} \mathrm{NK}$ cells was detected by flow cytometry. NK cells were seeded at $1 \times 10^{5}$ cells/well in a 24 -well plate, infected with the lentivirus at a multiplicity of infection (MOI) of 100 and supplemented with $8 \mu \mathrm{g} / \mathrm{ml}$ polybrene. At $6 \mathrm{~h}$, the NK cell culture medium was replaced with fresh medium.

Flow cytometric analysis. A single cell suspension of primary NK cells was prepared in Cell Staining Buffer (cat. no. 420201; BioLegend, Inc.). Cells were pre-incubated with $5 \mu$ of Human TruStain FcX ${ }^{\mathrm{TM}}$ (cat. no. 422301; BioLegend, Inc.) per $100 \mu \mathrm{l}$ ( $1 \times 10^{6}$ cells) of cell suspension for 5-10 min at room temperature to block Fc-receptors. The samples were centrifuged at $350 \mathrm{x}$ g for $5 \mathrm{~min}$ at room temperature and the supernatant was discarded. Cells were incubated with the following fluorescently labelled antibodies: Anti-CD3-FITC (5 $\mu \mathrm{l} / 1 \times 10^{6}$ cells; cat. no. 300305; BioLegend, Inc.) or anti-CD56-phycoerythrin (5 $\mu 1 / 1 \times 10^{6}$ cells; cat. no. 362507; BioLegend, Inc.) and incubated on ice for 15-20 min in the dark. Cells were washed two times with $2 \mathrm{ml}$ of Cell Staining Buffer and centrifuged at $350 \mathrm{x} \mathrm{g}$ for $5 \mathrm{~min}$ at room temperature. Cells were analyzed by flow cytometry using a BD FACSCalibur (Becton-Dickinson and Company) and data was analyzed using FCS Express 6.06.0022 (De Novo Software).
Western blot analysis. Western blotting was performed to examine c-MET expression in tumour cells and CAR expression in c-MET-CAR-NK cells. Briefly, cells were harvested and lysed with RIPA buffer (Beyotime Institute of Biotechnology) supplemented with a protease inhibitor cocktail and phenylmethylsulphonyl fluoride. Protein concentration was determined using the bicinchoninic acid method. A total of $20 \mu \mathrm{g}$ of the protein was loaded onto $7.5 \%$ SDS-PAGE at room temperature (RT). The proteins were transferred to PVDF membranes (Immobilon-P; EMD Millipore), blocked with $5 \%$ semi-skimmed milk for $1 \mathrm{~h}$ at RT and incubated overnight at $4^{\circ} \mathrm{C}$ with a primary anti-c-MET antibody (1:1,000; cat. no. AF1432; Beyotime Institute of Biotechnology) or anti-V5 antibody $(1: 1,000$; cat. no. 4AA261811F; Beijing 4A Biotech Co., Ltd.). The next day, the membranes were thoroughly rinsed with TBS $+0.05 \%$ Tween-20 and incubated for $1 \mathrm{~h}$ at RT with a horseradish peroxidase-conjugated secondary antibody (1:4,000; cat. no. A0218; Beyotime Institute of Biotechnology). Following mild stripping, the same membranes were incubated with a mouse anti- $\beta$-actin antibody $(1: 2,000$; cat. no. 3700T; Cell Signalling Technology, Inc.) for $1 \mathrm{~h}$ at room temperature as a loading control. Reaction products were detected using enhanced chemiluminescence (ECL Plus; GE Healthcare). ImageJ version K1.45 (National Institutes of Health) was used for densitometric analysis to quantify the relative expression of c-MET in the different cell lines.

Immunofluorescence. Immunostaining was performed as previously described (34). The staining reagents included an anti-c-MET antibody (1:500; cat. no. AF1432; Beyotime Institute of Biotechnology), Alexa Fluor 488-labelled goat anti-rabbit IgG antibody (1:400; cat. no. A0428; Beyotime Institute of Biotechnology), DAPI (cat. no. D9542; Sigma-Aldrich; Merck KGaA) and TRITC-conjugated phalloidin (1:1,000; cat. no. P1951; Sigma-Aldrich; Merck KGaA). The cells were imaged using an inverted fluorescent microscope (magnification, x20; Axio Observe 3; Carl Zeiss AG). Images were processed using ImageJ version K1.45.

Cytokine release assay. c-MET-CAR-NK, GFP-NK and NK cells were co-incubated with HepG2 or H1299 tumour cells at an effector-to-target (E:T) ratio of 2.5:1. Following $24 \mathrm{~h}$ of incubation at $37^{\circ} \mathrm{C}$, the supernatant was harvested and the concentration of released IFN- $\gamma$ was measured using the Human IFN- $\gamma$ ELISA kit (cat. no. SIF50; R\&D Systems, Inc.), according to the manufacturer's protocol.

In vitro cytotoxicity assay. Lactate dehydrogenase (LDH) release cytotoxicity assays were performed using the LDH Cytotoxicity Assay kit (Beyotime Institute of Biotechnology), following the manufacturer's protocol. c-MET-CAR-NK cells or NK cells and tumor cells (HEPG2 and H1299) were co-cultured at an E:T ratio of 2.5:1 for $4 \mathrm{~h}$ at $37^{\circ} \mathrm{C}$ in 96 -well plates in triplicate. Specific lysis was calculated as follows: Percentage of specific lysis=(experimental release-effector spontaneous release-target spontaneous release)/(target maximal release-target spontaneous release) x100\%.

Apoptosis assay. For apoptosis quantification, c-MET-CARNK cells or NK cells and tumor cells (HEPG2 and H1299) 
A

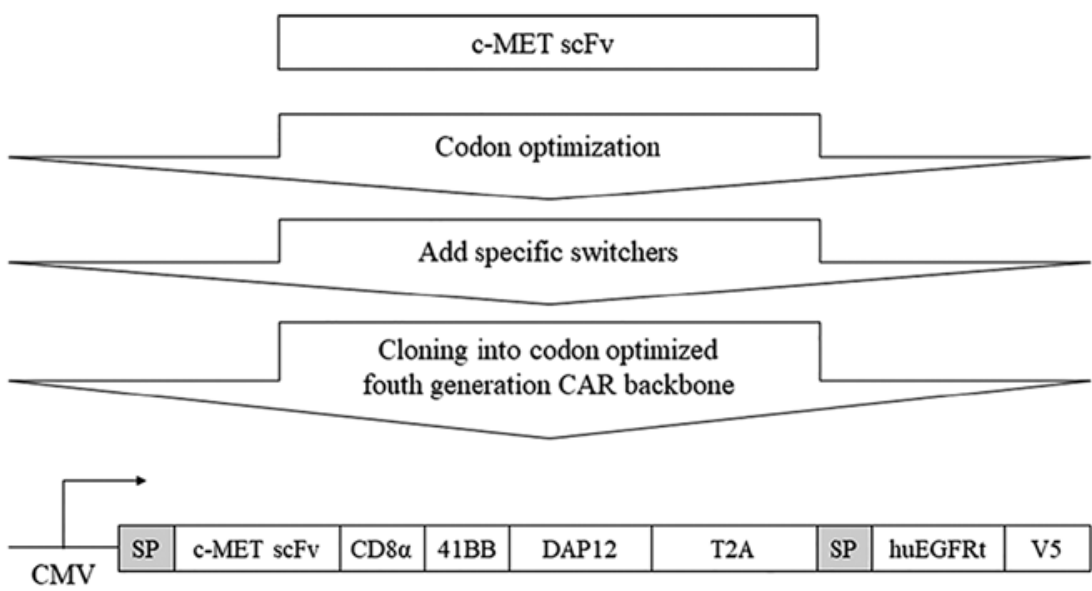

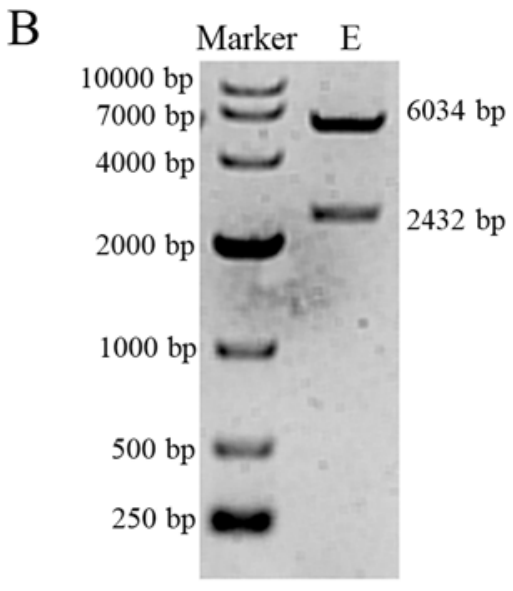

Figure 1. Construction of the c-MET-CAR lentiviral plasmid. (A) Schematic representation of the c-MET-CAR lentiviral construct, which consisted of an SP, C-MET-specific scFv antibody fragment and a CD8 $\alpha$ hinge region, a T2A self-cleaving peptide sequence followed by the intracellular domains of 41BB and DAP12, a signaling adaptor molecule involved in the signal transduction of the activating NK cell receptor. as well as a safety switch from huEGFRt. CAR expression was driven by the CMV promoter. (B) The constructed c-MET-CAR plasmid was digested with the restriction endonucleases KpnI and NotI and identified by agarose gel electrophoresis. The lengths of the two electrophoresis bands were 6,034 and 2,432 bp. SP, signal peptide; c-MET, c type proto-oncogene receptor tyrosine kinase; $\mathrm{CAR}$, chimeric antigen receptor; scFv, single-chain variable fragment; huEGFRt, truncated human epidermal growth factor receptor; CMV, cytomegalovirus; DAP12, TYRO protein tyrosine kinase-binding protein; E, experimental.

were co-cultured at an E:T ratio of $2.5: 1$ for $4 \mathrm{~h}$ at $37^{\circ} \mathrm{C}$ in 6-well plates in triplicate. The cells were washed twice with cold PBS and resuspend in $1 \mathrm{X}$ Binding Buffer (BD Biosciences) at a concentration of $1 \times 10^{5}$ cells $/ \mathrm{ml}$. A total of $5 \mu \mathrm{l}$ FITC Annexin V (BD Biosciences) and $5 \mu \mathrm{l}$ propidium iodide (BD Biosciences) were added, and the cells were gently vortexed and incubated for $15 \mathrm{~min}$ at $37^{\circ} \mathrm{C}$ in the dark. Subsequently, $400 \mu 11 \mathrm{X}$ Binding Buffer was added to each tube, and the cells were analysed by flow cytometry using a BD FACSCalibur (Becton-Dickinson and Company) within $1 \mathrm{~h}$ and data was analyzed using FCS Express 6.06.0022 (De Novo Software). All data are presented as the mean \pm standard deviation.

Live-cell video microscopy. A HepG2-mCherry stable cell line expressing the mCherry protein was created by transfection with the plasmid FT106-mCherry (gifts from Professor $\mathrm{Hu}$ Ying) using Lipofectamine ${ }^{\circledR} 3000$ reagent (Thermo Fisher Scientific, Inc.), according to the manufacturer's instructions. Stably transfected cells were selected using $600 \mu \mathrm{g} / \mathrm{ml}$ of G418 sulfate (Thermo Fisher Scientific, Inc.) for 3 weeks. The G418-resistant clones were isolated, expanded and maintained on plates in complete media with $400 \mu \mathrm{g} / \mathrm{ml} \mathrm{G} 418$ at $37^{\circ} \mathrm{C}$ in a humidified incubator with $5 \% \mathrm{CO}_{2}$. For live-cell video experiments, HepG2-mCherry cells $\left(5 \times 10^{3}\right.$ cells/well $)$ were plated in a 96-well plate and maintained at $37^{\circ} \mathrm{C}$ in a humidified incubator for $24 \mathrm{~h}$ to allow for adherence. Subsequently, c-MET-CAR-NK cells were added at a ratio of 1:1. The cells were imaged every $5 \mathrm{~min}$ for $36 \mathrm{~h}$ at $37^{\circ} \mathrm{C}$ using a Cytation1 imaging reader (BioTek Instruments, Inc.). Images were processed using ImageJ version K1.45.

Statistical analysis. Data are expressed as the mean \pm SD. Statistical analysis was performed with GraphPad Prism 5 software (GraphPad Software, Inc.). Student's t-test or one-way ANOVA with Bonferroni post hoc test were used to analyse the differences between groups. $\mathrm{P}<0.05$ was considered to indicate a statistically significant difference.

\section{Results}

Generation of the c-MET-CAR lentiviral plasmid. The scFv nucleotide sequence of an anti-c-MET antibody (Data S1A) was fused with a sequence encoding huEGFRt immediately following the V5 tag-encoding sequence. To improve signal transduction, the c-MET-CAR was designed with a CD8 $\alpha$ hinge and the intracellular signalling domains of 41BB and DAP12. The entire c-MET-scFv-CD8 $\alpha$-41BB-DAP12-EGFRt-V5 fragment (Fig. 1A) was ligated into a GFP lentiviral vector to construct the c-MET-CAR lentiviral plasmid. Fig. 1B shows bands at 6,034 and 2,432 bp after agarose gel electrophoresis of the digested recombinant c-MET-CAR lentiviral plasmid; the band sizes were consistent with the expected results. The sequencing results were consistent with the c-MET-CAR gene sequence, which suggested that the recombinant c-MET-CAR lentiviral plasmid was successfully constructed (Data S1B).

In vitro culture of human $N K$ cells induced from PBMCs. Human NK cells were induced from PBMCs isolated from fresh blood from healthy donors. Images of the cultured NK cells were taken on days 2, 14 and 21 under a microscope. Cell morphology changed from single small cells to large cell clusters (Fig. 2A). The growth curve demonstrated that the NK cells entered the rapid proliferation period on day 6 (Fig. 2B). The purity of the $\mathrm{CD}^{-} \mathrm{CD} 56^{+} \mathrm{NK}$ cells was detected by flow cytometry following 21 days of culture. In Fig. 2C, the upper left quadrant of the scatter diagram demonstrated that $>90 \%$ of the cells were $\mathrm{CD} 3{ }^{-} \mathrm{CD} 56^{+} \mathrm{NK}$ cells. The cytotoxicity of cultured NK cells was also tested on days 2, 14 and 21; the percentage of the NK cytotoxicity was 7.47, 25.93 and $27.40 \%$, respectively (Fig. 2D).

Generation of c-MET-CAR-NK cells. The c-MET-CAR lentiviral plasmid or control GFP lentiviral plasmid was co-transfected with the psPAX2 and pMD2.G packaging plasmids to produce the c-MET-CAR and GFP lentiviruses. To 
A
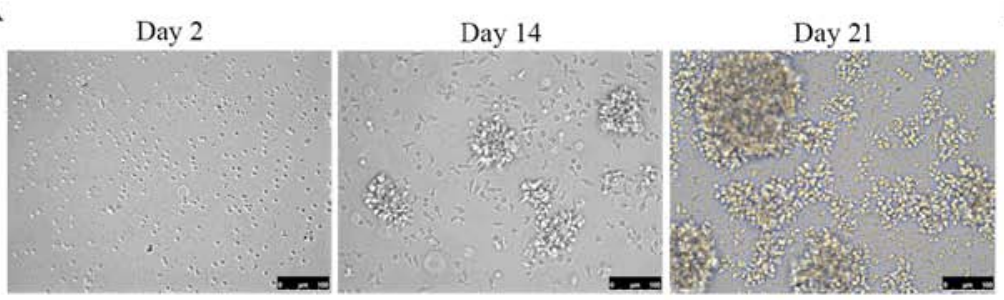

$\mathrm{C}$

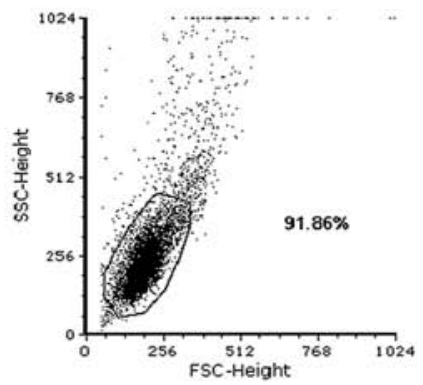

B

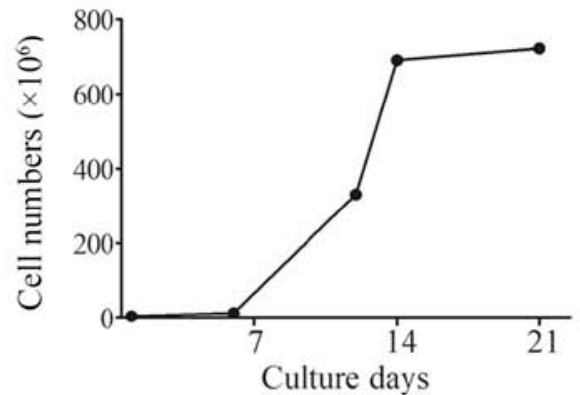

D

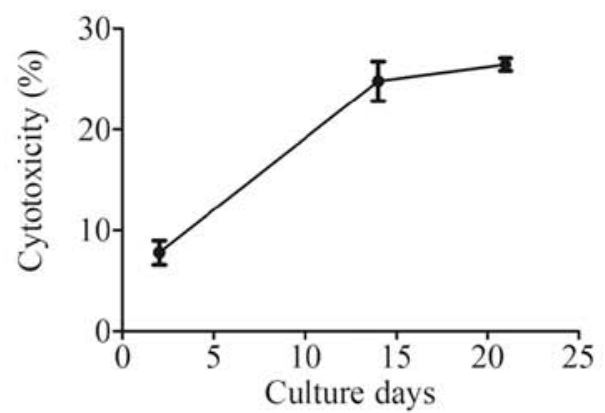

Figure 2. In vitro culture of human NK cells induced from peripheral blood mononuclear cells. (A) Images of cultured NK cells were taken on day 2, day 14 and day 21 under a microscope. Scale bar, $100 \mu \mathrm{m}$. (B) NK cell growth curve. (C) The purity of CD3-CD56 ${ }^{+}$NK cells was detected by flow cytometry after 17 days in culture. (D) NK cells were co-incubated with HepG2 tumour cells at an effector-to-target cell ratio of 2.5:1. Following 4 h of incubation, the cell lysis percentage was detected with a lactate dehydrogenase-release assay. NK, natural killer; FSC, forward scatter; SSC, side scatter.

confirm the titre, the c-MET-CAR lentivirus was diluted into six gradient concentrations and used to infect $293 \mathrm{~T}$ cells. After $48 \mathrm{~h}$ of infection, the infected $293 \mathrm{~T}$ cells were observed using fluorescence microscopy (Fig. 3A) and analysed for GFP expression using fluorescence-activated cell sorting (data not shown).

In vitro cultured human NK cells were transduced with the c-MET-CAR lentivirus or GFP lentivirus at the MOI of 100 to generate c-MET-CAR-NK cells or GFP-NK cells, respectively. The c-MET-CAR-NK cells were observed under brightfield (Fig. 3B, left) and a green channel filter (Fig. 3B, middle). According to the merged picture (Fig. 3B, right), $\sim 50 \%$ of the NK cells were GFP-positive. This subpopulation represented the c-MET-CAR-NK cells.

To validate the expression of c-MET-CAR in the transduced NK cells, western blot analysis was performed using an anti-V5 antibody that recognized the V5 tag within the c-MET-CAR structure. c-MET-CAR (V5) was expressed in the c-MET-CAR-NK cells, but not in the GFP-NK cells or normal NK cells (Fig. 3C).

c-MET is highly expressed in HepG2 cells but not H1299 cells. To assess the expression of c-MET in HepG2 and H1299 cells, cells were stained with a c-MET-specific antibody in an immunofluorescence assay (Fig. 4A). Fig. 4B demonstrates the statistical results of the fluorescence intensity in the green (c-MET) channel in Fig. 4A, indicating that the c-MET expression level in HepG2 cells was higher compared with that in the H1299 cells. The western blotting results further revealed that c-MET was highly expressed in the HepG2 cells, but expressed at a low level in the H1299 cells (Fig. 4C). HepG2 cell transfection with siRNA interfering with c-MET $\left(c-M E T^{R N A i}\right)$ revealed that the level of c-MET protein had been knocked down successfully (Fig. 4C, far left lane).
c-MET-CAR-NK cells kill HepG2 cells through c-MET $C A R$. Two cell lines were used to test the specificity of c-MET-CAR-NK cells: The c-MET low-expression cell line H1299 and c-MET high-expression cell line HepG2 (Fig. 5A). $c-M E T^{R N A i}$ HepG2 cells were used as a negative control. The E:T ratio was 2.5:1. The results demonstrated that the ratio of apoptotic cells in the H1299 group was 18.57, 18.87 and $19.54 \%$ for c-MET-CAR-NK, GFP-NK and NK, respectively. The ratio of apoptotic cells in the HepG2 group was 45.64, 20.74 and $16.86 \%$ for c-MET-CAR-NK, GFP-NK and NK, respectively. The ratio of apoptotic cells in $c-M E T^{R N A i}$ HepG2 cells group was $22.31,21.95$ and $18.31 \%$ for c-MET-CAR-NK, GFP-NK and NK, respectively. The cytotoxicity of c-MET-CAR-NK against the c-MET high-expression cell line HepG2 was significantly increased compared with the other two groups $(\mathrm{P}<0.01$; Fig. 5B).

The specific lysis of the two tumour cell lines induced by c-MET-CAR-NK cells was evaluated using the GFP-NK and normal NK cells as controls. The E:T ratio was 2.5:1. The cytotoxicity assay results demonstrated that c-MET-CAR-NK cells exhibited a significant increase in cytotoxicity against HepG 2 cells with a lysis ratio of $35.64 \pm 3.28 \%$ compared with the H1299 and $c$-MET ${ }^{R N A i}$ HepG2 groups (Fig. 5C), however, there was no significant difference when the E:T ratio was 5:1 (Fig. S1A).

The level of IFN- $\gamma$ secreted by c-MET-CAR-NK, GFP-NK and NK cells was determined by ELISA at an E:T ratio of 2.5:1; the concentration of IFN- $\gamma$ in the H1299 group was 882.36, 906.32 and $897.32 \mathrm{pg} / \mathrm{ml}$ in c-MET-CAR-NK, GFP-NK and NK, respectively. The concentration of IFN- $\gamma$ in the HepG2 group was $1,288.35,787.67$ and $908.25 \mathrm{pg} / \mathrm{ml}$ for c-MET-CAR-NK, GFP-NK and NK, respectively. The concentration of IFN- $\gamma$ in $c-M E T^{R N A i}$ HepG2 group was 940.17, 918.25 and $962.23 \mathrm{pg} / \mathrm{ml}$ 


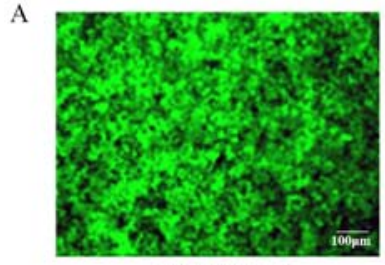

Undiluted

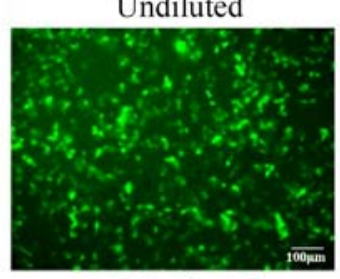

$1 / 64$ diluted

B

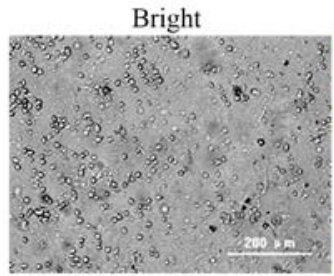

$\mathrm{C}$

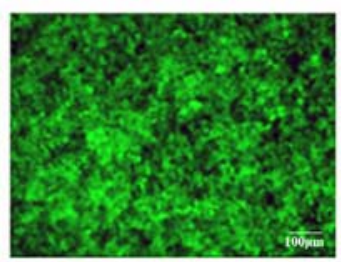

$1 / 4$ diluted

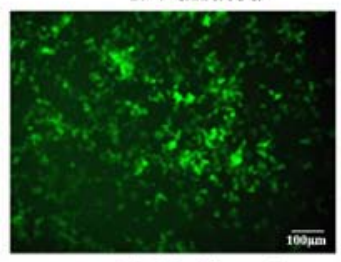

$1 / 128$ diluted
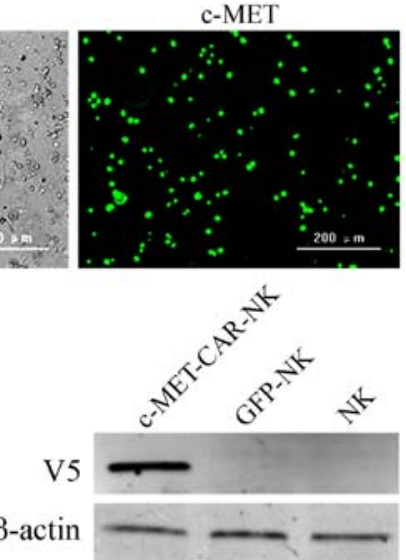

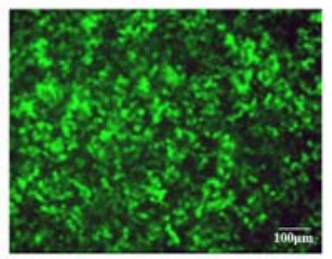

$1 / 16$ diluted

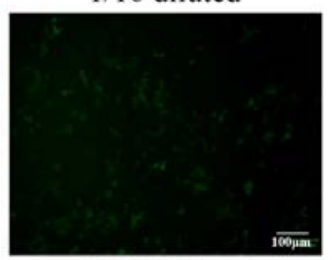

$1 / 256$ diluted

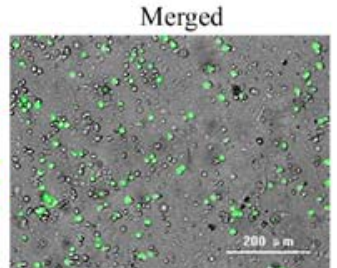

Figure 3. Generation of c-MET-CAR-NK cells. (A) The c-MET-CAR lentiviral titre was calculated by diluting the lentivirus and infecting $293 \mathrm{~T}$ cells for $72 \mathrm{~h}$. Scale bar, $100 \mu \mathrm{m}$. (B) In vitro cultured human NK cells were infected with the c-MET-CAR lentivirus to generate c-MET-CAR-NK cells. Scale bar, $200 \mu \mathrm{m}$. (C) Western blot analysis was used to assess the c-MET-CAR expression in NK, GFP-NK and c-MET-CAR-NK cells. The expression of c-MET-CAR was detected with a specific anti-V5 antibody. $\beta$-actin was used as loading control. NK, natural killer; c-MET, c type proto-oncogene receptor tyrosine kinase; CAR, chimeric antigen receptor; GFP, green fluorescent protein.

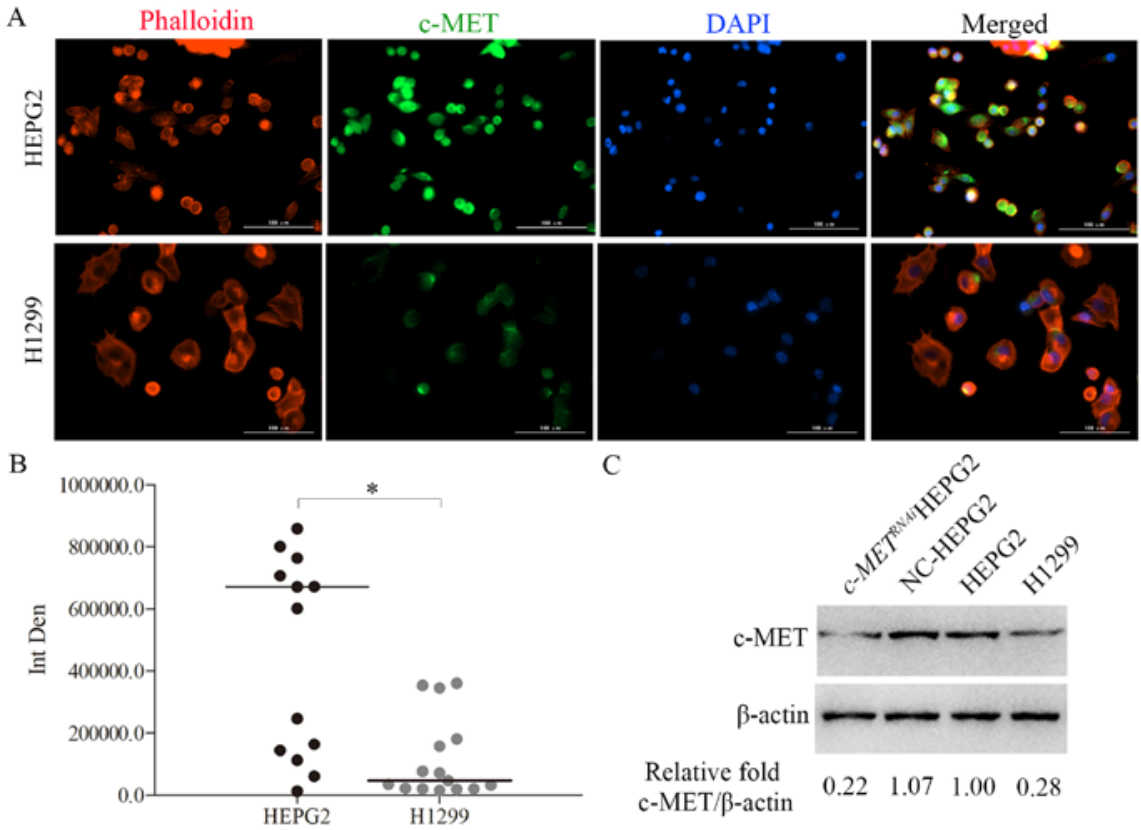

Figure 4. c-MET is highly expressed in HepG2 cells, but not H1299 cells. (A) Immunofluorescence staining with an anti-c-MET antibody (green), phalloidin (red) and DAPI (blue) in HepG2 cells (top) and H1299 cells (bottom). Scale bar, $100 \mu \mathrm{m}$. (B) Fluorescence intensity of the green (c-MET) channel in the immunofluorescence images above. "P<0.05. (C) Western blotting of the relative c-MET protein expression levels in $c-M E T^{\text {RNAi }}$ HEPG2, NC-HEPG2, HepG2 and $\mathrm{H} 1299$ cells. $\beta$-actin was used as loading control. c-MET, c type proto-oncogene receptor tyrosine kinase; $c-M E T^{R N A i}$, cells treated with small interfering RNA targeting c-MET; NC, negative control; Int Den, fluorescence intensity. 

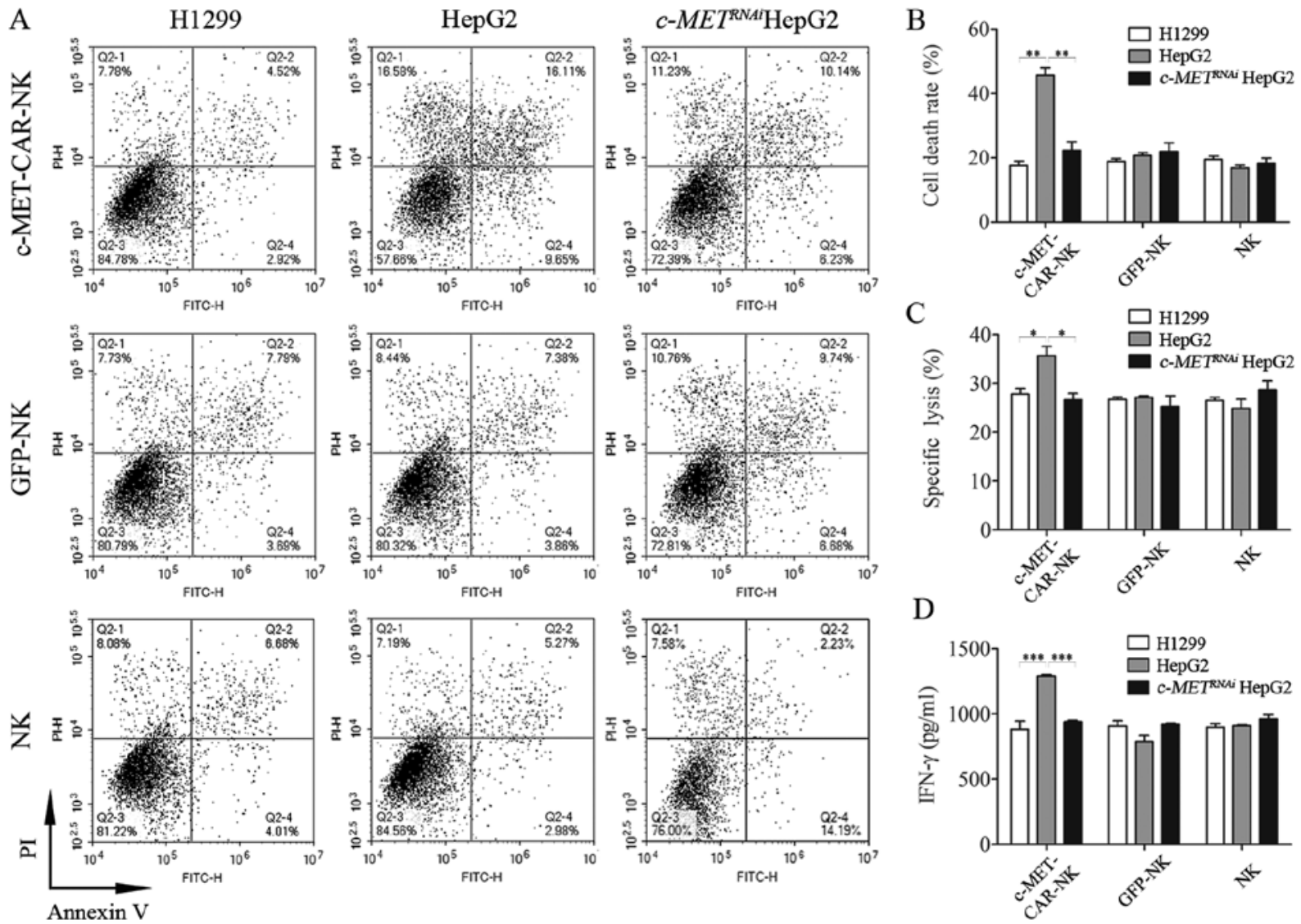

Figure 5. c-MET-CAR-NK cells specifically kill HepG2 cells. c-MET-CAR-NK, GFP-NK and NK cells were co-incubated with H1299, HepG2 or $c$-MET ${ }^{R N A i}$ HepG2 cells at an effector-to-target ratio of 2.5:1. After $4 \mathrm{~h}$ of incubation, apoptosis was (A) measured by flow cytometry and (B) quantified. (C) After $4 \mathrm{~h}$ of incubation, the specific lysis percentage was detected with a lactate dehydrogenase release assay. (D) After $24 \mathrm{~h}$ of incubation, the supernatant was harvested and the concentration of the released IFN- $\gamma$ was measured with a sandwich ELISA. The data are presented as the mean \pm SEM from three independent experiments. ${ }^{*} \mathrm{P}<0.05,{ }^{* *} \mathrm{P}<0.01$ and ${ }^{* * * *} \mathrm{P}<0.001$. NK, natural killer; c-MET, c type proto-oncogene receptor tyrosine kinase; CAR, chimeric antigen receptor; GFP, green fluorescent protein; IFN- $\gamma$, interferon- $\gamma ; c-M E T^{R N A i}$, cells treated with short interfering RNA targeting c-MET; PI, propidium iodide.

for c-MET-CAR-NK, GFP-NK and NK, respectively. The level of IFN- $\gamma$ secreted by c-MET-CAR-NK against the c-MET high-expression cell line HepG2 was significantly increased compared with the other two groups $(\mathrm{P}<0.001$; Fig. $5 \mathrm{D})$, however, there was no significant difference when the E:T ratio was 5:1 (Fig. S1B).

Observation of c-MET-CAR-NK cell cytotoxicity in vitro using live imaging. The cytotoxic activity of c-MET-CAR-NK cells against HepG2 cells stably expressing mCherry (HepG2-mCherry) was analysed using live imaging. The integrated process of a mitotic HepG 2 cell being attacked by a c-MET-CAR-NK cell was recorded and analysed (Fig. 6). The mCherry fluorescence gradually disappeared, representing the apoptosis of a c-MET-positive HepG2 cell, which was due to the attack from c-MET-CAR-NK cells (Fig. 6A). The quantification of the live imaging results is presented in Fig. 6B. Following 30 min of c-MET-CAR-NK and HepG2 cell co-culture, the fluorescence intensity of HepG2 cell reduced from 25 to 6.5 mean grey value, which indicated the death of the target cells.

\section{Discussion}

The present study focused on the possibility of developing a specific cell therapy using NK cells as effector cells in HCC.
Based on previous in vitro data, c-MET was been identified as a carcinogen in liver cancer (17-19). Consequently, a CAR structure that can guide NK cells targeting the c-MET antigen expressed on the HCC cell surface was constructed in the present study. The results demonstrated a high affinity between c-MET-CAR-positive NK cells and HepG2 liver cancer cells. Based on these results, CAR-NK cell-based immunotherapy may provide a safe and specific approach for liver cancer therapy. However, the exact curative effect of CAR-NK cell immunotherapy still needs to be demonstrated in animal models and clinical trials. Apart from NK cell cytotoxicity, cytokines such as IFN- $\gamma$ are involved in the effect of NK cell-based immunotherapy. NK cells are an early producer of IFN- $\gamma$, which exerts multiple effects on the immune response, such as the induction of major histocompatibility complex class II molecules on antigen-presenting cells, activation of myeloid cells and induction of T helper 1 cells, as well as angiogenesis. Macrophage activation by NK cell-derived IFN- $\gamma$ has been demonstrated to be essential for the resistance to chemical carcinogenesis in a mouse model of primary tumorigenesis. Thus, IFN- $\gamma$ is an important anti-tumour cytokine (35).

In the majority of published studies, the NK92 cell line was used as effective cells against tumours $(3,4,36)$. In the present study, a method to produce autologous NK cells from PBMCs was developed. The advantage of this method is that it guarantees 
A
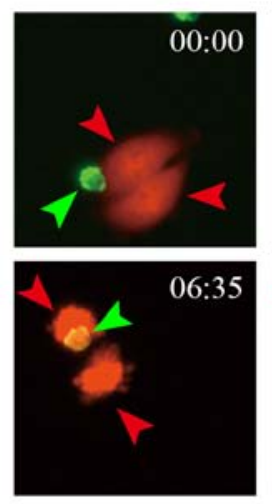
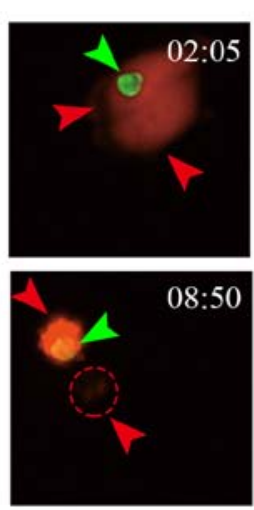
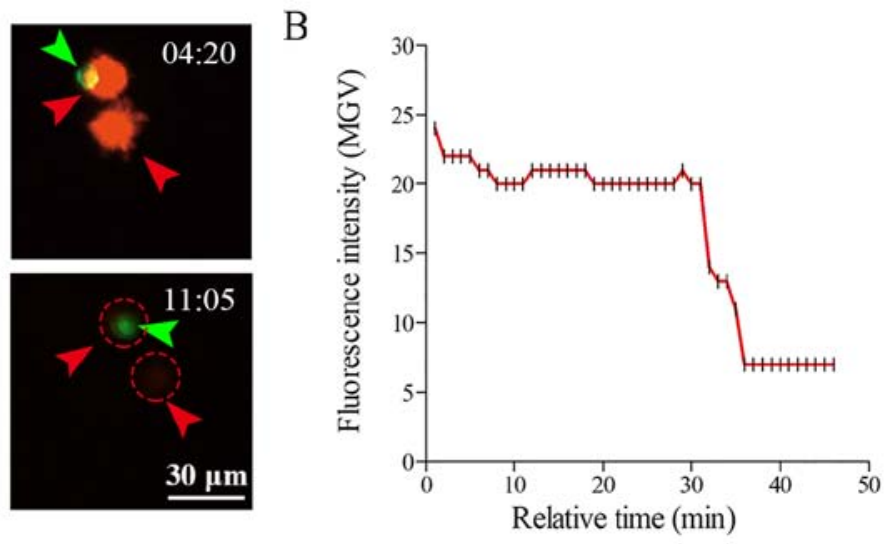

Figure 6. Observation of c-MET-CAR-NK cell cytotoxicity in vitro using live imaging. (A) Frames of a time-lapse video demonstrating the process of one c-MET-CAR-NK cell (green, green arrow) killing HepG2 cells (red, red arrows). Scale bar, $30 \mu \mathrm{m}$. (B) The MGV of the fluorescence intensity of HepG2 cells (red channel) in the time-lapse video above was calculated. NK, natural killer; c-MET, c type proto-oncogene receptor tyrosine kinase; CAR, chimeric antigen receptor; MGV, mean grey value.

safety for potential future clinical applications. In addition, a lentivirus system was adapted to transduce the c-MET-CAR structure into effective NK cells, which largely increased the ratio of infected NK cells from the $30 \%$ reported by previous studies $(19,37,38)$ to $>50 \%$. In addition, c-Met-CAR-engineered NK cells were more specific and cytotoxic against the liver cancer cell line HepG2 with high c-MET expression compared with the lung cancer cell line H1299 with low c-MET expression. These results suggest that CAR-NK cells may be a promising, specific and safe approach for liver cancer treatment.

Although the preliminary data of the present study demonstrated that c-MET-CAR-NK had potential application prospects in liver cancer, several problems emerged during the development process. Firstly, it has been reported in another study that the lifespan of CAR-NK cell is only 28 days in vivo (39), which may result in a reduction of persistence of the curative effect due to the high cost of CAR-NK cells. Secondly, the dose of CAR-NK cells may be difficult to apply in vivo. Previous studies have reported the optimal anti-tumour activity for CAR-NK cells in vivo, which is $1 \times 10^{7}$ cells/mouse $(40,41)$. However, the titer of the lentivirus in the present study was MOI=100. Lentiviruses are used as a gene-modification tool in gene therapy. The first lentiviral therapy, Tisagenlecleucel, was approved in the United States in 2017 for the treatment of pediatric and young adult patients with acute lymphoblastic leukemia (42). The safety of lentiviruses has been accepted worldwide (43).

There are also limitations to solid tumour treatment using CAR-NK cells. The most concerning problem is the off-target effect caused by tumour-associated antigen mutation post-CAR-NK treatment, as similar problems have been reported in CAR-T therapy (36,38). Recently, Zhang et al (37) reported that the checkpoint receptor $\mathrm{T}$ cell immunoreceptor with immunoglobulin and immunoreceptor tyrosine-based inhibitory motif domains was associated with NK cell exhaustion in tumour-bearing mice and patients with colon cancer. Thus, the microenvironment of solid tumours may inhibit the anti-tumour activity of CAR-NK cells. However, potential NK cell immune checkpoint blockers such as anti-programmed cell death protein 1 antibody for $\mathrm{T}$ cells may be used as a combined treatment in future studies.

\section{Acknowledgements}

The authors would like to thank Professor Hu Ying of The Harbin Institute of Technology for providing the psPAX2 and pMD2.G plasmids.

\section{Funding}

The present study was supported by the Shenzhen Science and Technology Innovation Committee (grant nos. KQJSCX20170331160008397, JCYJ20170412155231633, JCYJ20170307171034705, JCYJ20170816105345191 and GGFW2016030117123665) and the Science and Technology Planning Project of Shenzhen, China (grant no. JCYJ20170816105345191).

\section{Availability of data and materials}

The datasets used and analysed during the current study are available from the corresponding author on reasonable request.

\section{Authors' contributions}

BL and ZZL performed the experiments. MLZ, JWL, XMC, WBG, ZL and ZDY assisted with the experiments. TL designed the project and wrote the manuscript.

\section{Ethics approval and consent to participate}

The generation of human NK cells was approved by the Ethics Committee of Shenzhen Luohu People's Hospital. All blood donors provided written informed consent for the collection of samples and subsequent analyses.

\section{Patient consent for publication}

Not applicable.

\section{Competing interests}

The authors declare that they have no competing interests. 


\section{References}

1. Yuan P, Chen TH, Chen ZW and Lin XQ: Calculation of life-time death probability due malignant tumors based on a sampling survey area in China. Asian Pac J Cancer Prev 15: 4307-4309, 2014.

2. Omata M, Cheng AL, Kokudo N, Kudo M, Lee JM, Jia J, Tateishi R, Han KH, Chawla YK, Shiina S, et al: Asia-Pacific clinical practice guidelines on the management of hepatocellular carcinoma: A 2017 update. Hepatol Int 11: 317-370, 2017.

3. Ma W, Wu L, Zhou F, Hong Z, Yuan Y and Liu Z: T cell-associated immunotherapy for hepatocellular carcinoma. Cell Physiol Biochem 41: 609-622, 2017.

4. Wang Z, Wu Z, Liu Y and Han W: New development in CAR-T cell therapy. J Hematol Oncol 10: 53, 2017.

5. DeFrancesco L: CAR-T cell therapy seeks strategies to harness cytokine storm. Nat Biotechnol 32: 604, 2014.

6. Klingemann H: Are natural killer cells superior CAR drivers? Oncoimmunology 3: e28147, 2014.

7. Glienke W, Esser R, Priesner C, Suerth JD, Schambach A, Wels WS, Grez M, Kloess S, Arseniev L and Koehl U: Advantages and applications of CAR-expressing natural killer cells. Front Pharmacol 6: 21, 2015.

8. Bordon Y: Tumour immunology: Natural killer cells spy greedy tumours. Nat Rev Immunol 18: 77, 2018.

9. Rosenberg EB, Herberman RB, Levine PH, Halterman RH, McCoy JL and Wunderlich JR: Lymphocyte cytotoxicity reactions to leukemia-associated antigens in identical twins. Int J Cancer 9: 648-658, 1972.

10. Qian X, Wang X and Jin H: Cell transfer therapy for cancer: Past, present, and future. J Immunol Res 2014: 525913, 2014

11. Vivier E, Raulet DH, Moretta A, Caligiuri MA, Zitvogel L, Lanier LL, Yokoyama WM and Ugolini S: Innate or adaptive immunity? The example of natural killer cells. Science 331: 44-49, 2011.

12. Bradley M, Zeytun A, Rafi-Janajreh A, Nagarkatti PS and Nagarkatti M: Role of spontaneous and interleukin-2-induced natural killer cell activity in the cytotoxicity and rejection of Fas+ and Fas- tumor cells. Blood 92: 4248-4255, 1998.

13. Screpanti V, Wallin RP, Ljunggren HG and Grandien A: A central role for death receptor-mediated apoptosis in the rejection of tumors by NK cells. J Immunol 167: 2068-2073, 2001

14. Kayagaki N, Yamaguchi N, Nakayama M, Takeda K, Akiba H, Tsutsui H, Okamura H, Nakanishi K, Okumura K and Yagita H: Expression and function of TNF-related apoptosis-inducing ligand on murine activated NK cells. J Immunol 163: 1906-1913, 1999.

15. Brehm C, Huenecke S, Esser R, Kloess S, Quaiser A, Betz S Zimmermann O, Soerensen J, Passweg JR, Klingebiel T, et al: Interleukin-2-stimulated natural killer cells are less susceptible to mycophenolate mofetil than non-activated NK cells: possible consequences for immunotherapy. Cancer Immunol Immunother 63 : 821-833, 2014

16. Campbell KS and Hasegawa J: Natural killer cell biology: An update and future directions. J Allergy Clin Immunol 132: 536-544, 2013

17. Guillerey C, Huntington ND and Smyth MJ: Targeting natural killer cells in cancer immunotherapy. Nat Immunol 17: 1025-1036, 2016

18. Rezvani K, Rouce R, Liu E and Shpall E: Engineering natural killer cells for cancer immunotherapy. Mol Ther 25: 1769-1781, 2017.

19. Mehta RS and Rezvani K: Chimeric antigen receptor expressing natural killer cells for the immunotherapy of cancer. Front Immunol 9: 283, 2018.

20. Fasolo A, Sessa C, Gianni L and Broggini M: Seminars in clinical pharmacology: An introduction to MET inhibitors for the medical oncologist. Ann Oncol 24: 14-20, 2013.

21. Furge KA, Zhang YW and Vande Woude GF: Met receptor tyrosine kinase: Enhanced signaling through adapter proteins. Oncogene 19: 5582-5589, 2000.

22. Ma PC, Maulik G, Christensen J and Salgia R: c-Met: Structure, functions and potential for therapeutic inhibition. Cancer Metastasis Rev 22: 309-325, 2003.

23. Zhang YW, Su Y, Volpert OV and Vande Woude GF: Hepatocyte growth factor/scatter factor mediates angiogenesis through positive VEGF and negative thrombospondin 1 regulation. Proc Natl Acad Sci USA 100: 12718-12723, 2003.

24. Zhuang PH, Xu L, Gao L, Lu W, Ruan LT and Yang J: Correlations of microvascular blood flow of contrast-enhanced ultrasound and HGF/c-Met signaling pathway with clinicopathological features and prognosis of patients with hepatocellular carcinoma. Onco Targets Ther 10: 847-857, 2017.
25. Bouattour M, Raymond E, Qin S, Cheng AL, Stammberger U, Locatelli G and Faivre S: Recent developments of c-Met as a therapeutic target in hepatocellular carcinoma. Hepatology 67: $1132-1149,2018$

26. Kim JH, Kim HS, Kim BJ, Jang HJ and Lee J: Prognostic value of c-Met overexpression in hepatocellular carcinoma: A meta-analysis and review. Oncotarget 8: 90351-90357, 2017.

27. Yan S, Jiao X, Zou H and Li K: Prognostic significance of c-Met in breast cancer: A meta-analysis of 6010 cases. Diagn Pathol 10: 62, 2015.

28. Pyo JS, Kang G, Cho WJ and Choi SB: Clinicopathological significance and concordance analysis of c-MET immunohistochemistry in non-small cell lung cancers: A meta-analysis. Pathol Res Pract 212: 710-716, 2016.

29. Liu Y, Yu XF, Zou J and Luo ZH: Prognostic value of c-Met in colorectal cancer: A meta-analysis. World J Gastroenterol 21: 3706-3710, 2015.

30. Xin Y, Jin D, Eppler S, Damico-Beyer LA, Joshi A, Davis JD, Kaur S, Nijem I, Bothos J, Peterson A, et al: Population pharmacokinetic analysis from phase I and phase II studies of the humanized monovalent antibody, onartuzumab (MetMAb), in patients with advanced solid tumors. J Clin Pharmacol 53: 1103-1111, 2013.

31. Lohitesh K, Chowdhury R and Mukherjee S: Resistance a major hindrance to chemotherapy in hepatocellular carcinoma: An insight. Cancer Cell Int 18: 44, 2018.

32. Zhang Q, Zhou M, Wu X, Li Z, Liu B, Gao W, Yue J and Liu T: Promoting therapeutic angiogenesis of focal cerebral ischemia using thrombospondin-4 (TSP4) gene-modified bone marrow stromal cells (BMSCs) in a rat model. J Trans Med 17: 111, 2019

33. Gandara C, Affleck V and Stoll EA: Manufacture of thirdgeneration lentivirus for preclinical use, with process development considerations for translation to good manufacturing practice. Hum Gene Ther Methods 29: 1-15, 2018.

34. Wang YF, Kunda PE, Lin JW, Wang H, Chen XM, Liu QL and Liu T: Cytokine-induced killer cells co-cultured with complete tumor antigen-loaded dendritic cells, have enhanced selective cytotoxicity on carboplatin-resistant retinoblastoma cells. Oncol Rep 29: 1841-1850, 2013.

35. Jackson HJ and Brentjens RJ: Overcoming antigen escape with CAR T-cell therapy. Cancer Discov 5: 1238-1240, 2015.

36. Harjes U: CAR antigens beyond recognition. Nat Rev Cancer 18: 723,2018 .

37. Zhang Q, Bi J, Zheng X, Chen Y, Wang H, Wu W, Wang Z, Wu Q, Peng $\mathrm{H}$, Wei $\mathrm{H}$, et al: Blockade of the checkpoint receptor TIGIT prevents NK cell exhaustion and elicits potent anti-tumor immunity. Nature immunology 19: 723-732, 2018.

38. Orlando EJ, Han X, Tribouley C, Wood PA, Leary RJ, Riester M, Levine JE, Qayed M, Grupp SA, Boyer M, et al: Genetic mechanisms of target antigen loss in CAR19 therapy of acute lymphoblastic leukemia. Nat Med 24: 1504-1506, 2018.

39. Li Y, Hermanson DL, Moriarity BS and Kaufman DS: Human iPSC-derived natural killer cells engineered with chimeric antigen receptors enhance anti-tumor activity. Cell Stem Cell 23: 181-192 e185, 2018.

40. Oelsner S, Waldmann A, Billmeier A, Röder J, Lindner A, Ullrich E, Marschalek R, Dotti G, Jung G, Grosse-Hovest L, et al: Genetically engineered CAR NK cells display selective cytotoxicity against FLT3-positive B-ALL and inhibit in vivo leukemia growth. Int J Cancer: Mar 13, 2019 (Epub ahead of print).

41. Shiozawa M, Chang CH, Huang YC, Chen YC, Chi MS, Hao HC, Chang YC, Takeda S, Chi KH and Wang YS: Pharmacologically upregulated carcinoembryonic antigen-expression enhances the cytolytic activity of genetically-modified chimeric antigen receptor NK-92MI against colorectal cancer cells. BMC Immunol 19: 27, 2018.

42. Escors D and Breckpot K: Lentiviral vectors in gene therapy: Their current status and future potential. Arch Immunol Ther Exp (Warsz) 58: 107-119, 2010.

43. Schambach A, Zychlinski D, Ehrnstroem B and Baum C: Biosafety features of lentiviral vectors. Hum Gene Ther 24: $132-142,2013$.

This work is licensed under a Creative Commons Attribution-NonCommercial-NoDerivatives 4.0 International (CC BY-NC-ND 4.0) License. 